\title{
Activities for 1030/1090 MHz Spectrum Saturation Alleviation
}

\author{
Stanislav Pleninger \\ Department of Air Transport \\ Czech Technical University in Prague \\ Prague, Czech Republic \\ pleninger@fd.cvut.cz
}

\author{
Miloš Strouhal \\ Department of Air Transport \\ Czech Technical University in Prague \\ Prague, Czech Republic \\ strouhal@fd.cvut.cz
}

\begin{abstract}
The paper describes the danger of continuing inevitable growing of transmissions on 1030/1090 MHz RF band and actual efforts for alleviation of $\mathrm{RF}$ pollution in the light of development of new technologies.
\end{abstract}

Keywords-component; SSR, Mode S, ACAS, 1090 ES, Extended Squitter, Hybride Surveillance, Phase Modulation replies

\section{INTRODUCTION}

1030 and $1090 \mathrm{MHz}$ remain the worldwide RF bands used to support cooperative aeronautical surveillance systems. These bands are shared by different applications such as SSR Mode A/C, SSR Mode S, MLAT (Multilateration Systems), ACAS, ADS-B 1090ES (1090 MHz Extended Squitter), including military applications. The global use of these RF bands allows a cost effective interoperable system [8].

On the other hand sharing this one spectrum among all above mentioned systems take the RF band very congested in high density airspace. The predicted growth in air traffic and an increased use of the aeronautical surveillance spectrum by both the civil and military surveillance communities creates the possibility that sometimes in the future, the spectrum may become congested to a point that existing and planned aeronautical surveillance and collision avoidance systems may not meet their desired performance requirements [1].

Many activities have been realized in order to improve spectrum efficiency during last decade. Significant optimization of 1030/1090 MHz band was achieved through the use of:

- SSR Mode S that removed the old SSR sliding window based systems.

- The reduction in the use of active radars due to installation of passive MLAT systems in cooperation with implementation of ADS-B technology.

- Through radar clustering and increase data sharing between surveillance service providers (civil/civil and civil/military) and improved management of Mode $\mathrm{S}$ interrogators.

- ACAS interrogating algorithms modification (i.e. hybrid surveillance, ...)

- Increasing the capacity of 1090ES using a phase modulation technique

In particular last two points are nowadays subjected to intensive investigation, specification and standardization process within the scope of ICAO. There are in more details introduced in later parts in this paper.

\section{1030/1090 RF BAND MEASUREMENT IN EUROPE}

Real measurements were carried out in order to detailed assessment of the actual situation in European high traffic density areas. Figures below summarized the records achieved during the measurements campaign in 2011 in Europe under the auspices of EUROCONTROL by means of equipment installed on Airbus Beluga aircrafts. The modified TCAS receiver Collins TTR 920 was utilized to monitoring and recording signal on 1090 RF band (receiver sensitivity level $84 \mathrm{dBm}$ ) and modified Mode $\mathrm{S}$ transponder which enable 1030 $\mathrm{RF}$ band recording (receiver sensitivity level $-70 \mathrm{dBm}$ ). Data were recorded and subsequently analyzed off-line. The results were presented at different places in Europe, and in relation with the subsequent pictures the areas are specified by the following waypoints:
AMVEL: $53.18417^{\circ} \mathrm{N}, 0.03194^{\circ} \mathrm{W}$
BASUM: $52.77199^{\circ} \mathrm{N}, 8.78854^{\circ} \mathrm{W}$
BOMBI: $50.05667^{\circ} \mathrm{N}, 8.80028^{\circ} \mathrm{E}$
HELEN: $51.23531^{\circ} \mathrm{N}, 3.86971^{\circ} \mathrm{E}$
KENUM: $50.7225^{\circ} \mathrm{N}, 6.23944^{\circ} \mathrm{E}$
KUDES: $47.39456^{\circ} \mathrm{N}, 8.63522^{\circ} \mathrm{E}$
LFOB: $49.45444^{\circ} \mathrm{N}, 2.11278^{\circ} \mathrm{E}$
MOLUS: $46.44389^{\circ} \mathrm{N}, 6.67944^{\circ} \mathrm{E}$
ROLEN: $48.58^{\circ} \mathrm{N}, 0.15667^{\circ} \mathrm{E}$
TALGA: $51.95833^{\circ} \mathrm{N}, 3.24556^{\circ} \mathrm{E}$ 


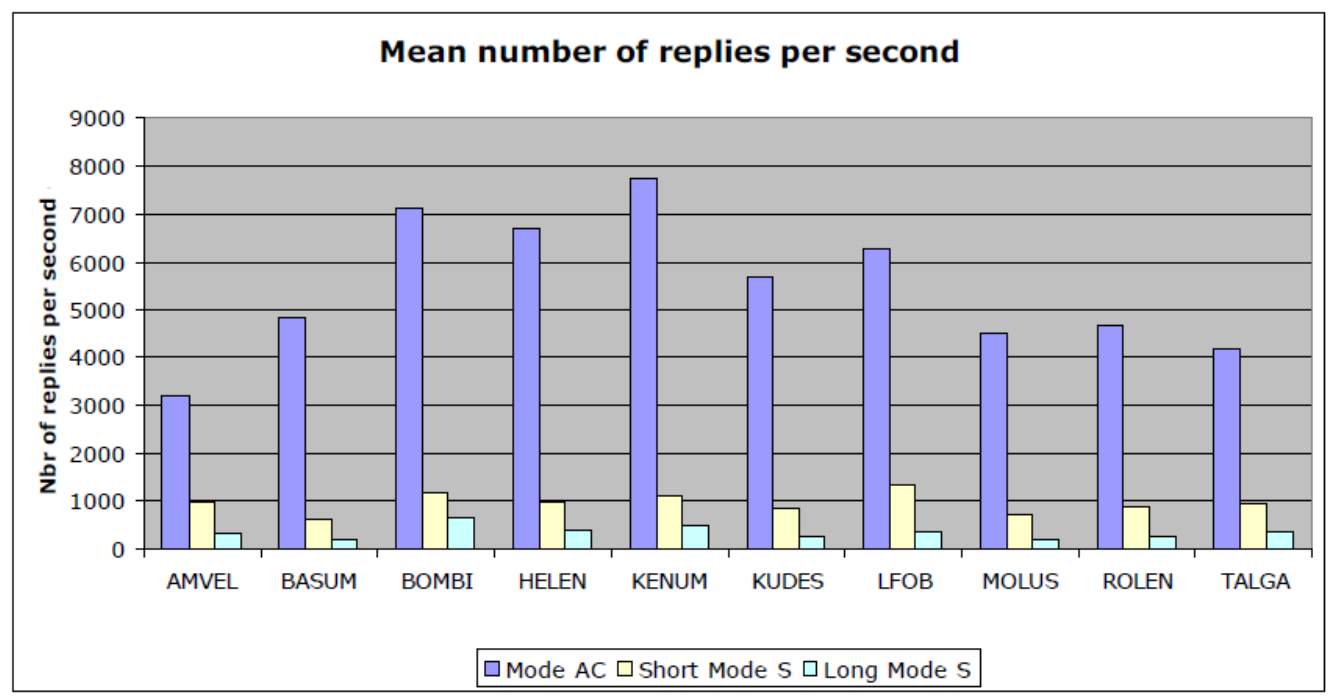

Figure 1. Mean number of replies per second on $1090 \mathrm{MHz}$ frequency band [2].

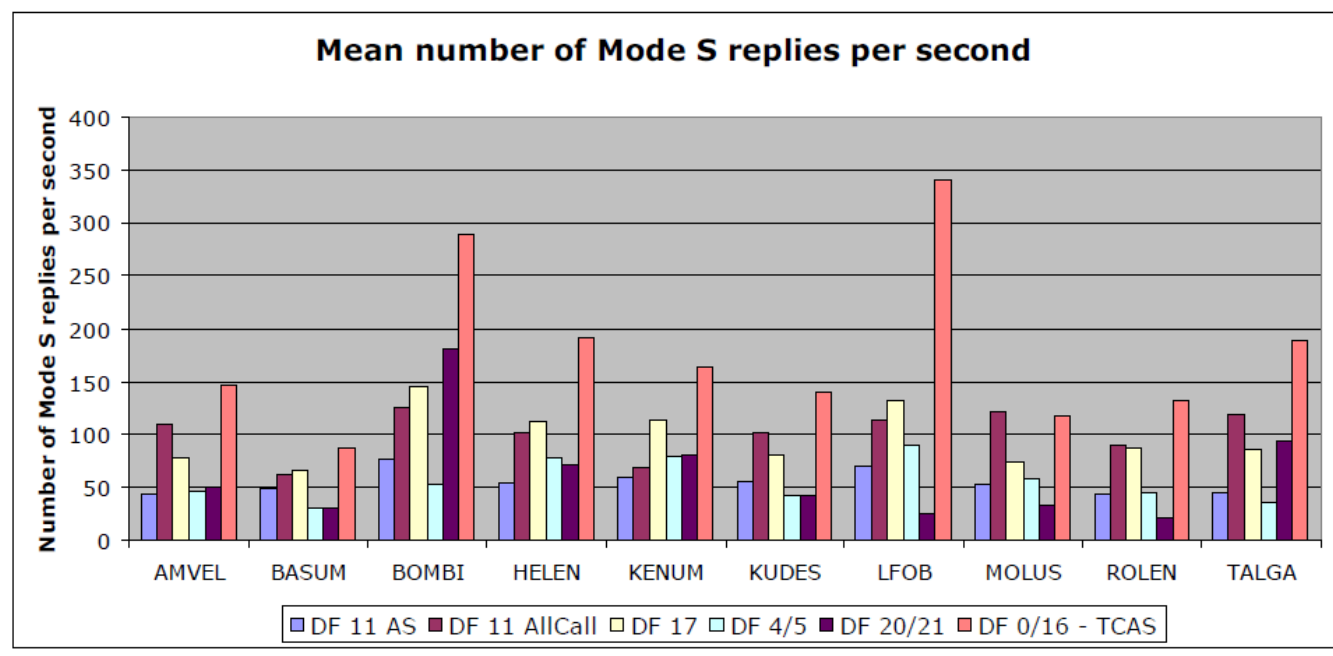

Figure 2. Mean number of Mode S replies per second on $1090 \mathrm{MHz}$ frequency band [2].

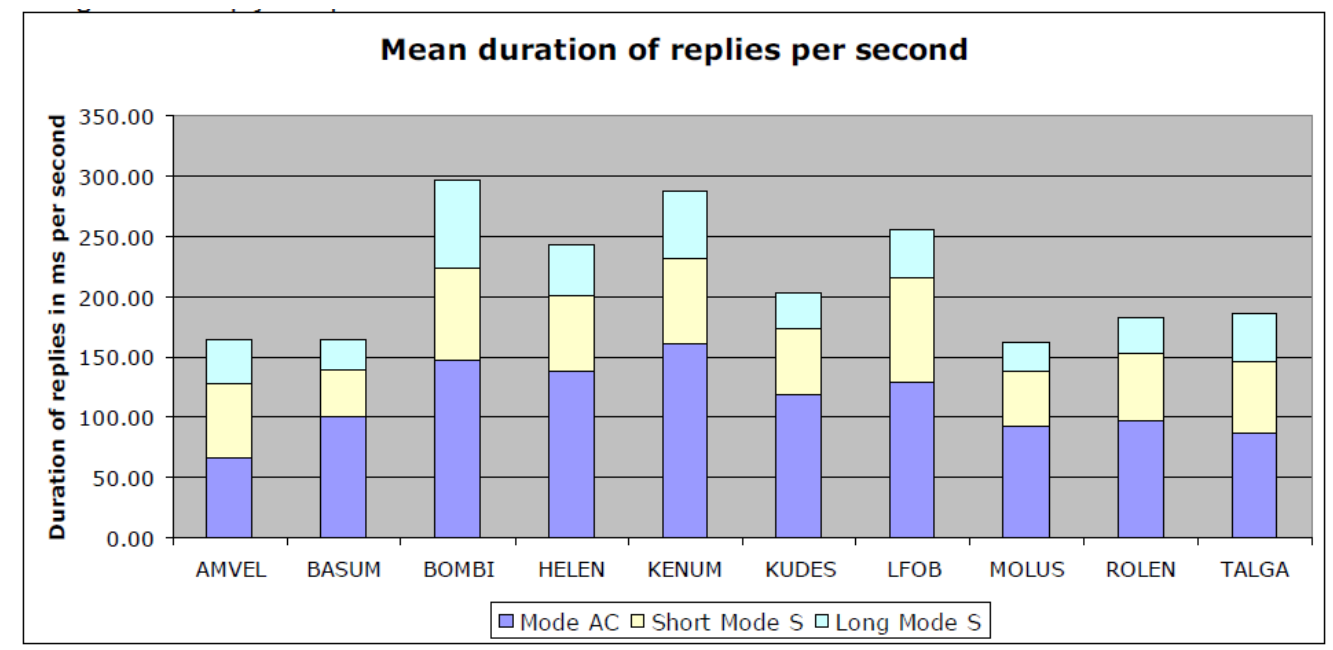

Figure 3. Mean duration of replies per second on $1090 \mathrm{MHz}$ frequency band [2]. 
Different types of reply have different duration (Mode A/C reply: $20.75 \mu \mathrm{s}$, short Mode S reply: $64 \mu \mathrm{s}$, long Mode S reply: $120 \mu \mathrm{s})$. This aspect must be taken into account for further analysis. This is evident if we compare Fig 3 with Fig. 1. On Fig. 1 you can see dominant mode $\mathrm{A} / \mathrm{C}$ replays, whereas on the Fig. 3 where overall duration of Mode A/C replies to Mode S (short and long) replies are almost equal in many regions.

Besides of global load view (i.e. measurement of all transmission on $1090 \mathrm{MHz}$ ) very interesting aspect is the loading from one Mode $\mathrm{S}$ transponder perspective. The statistic presented below represents real recorded peak transmission value from measurement on Beluga aircraft [6].

$$
\begin{aligned}
& \text { DF11 squitter }(\mathrm{II}=0)=1 \text { message } / \mathrm{s} \\
& \text { DF11 }(\mathrm{II} \neq 0)=20 \text { messages } / \mathrm{s} \\
& \text { DF4/5= } 2 \text { messages } / \mathrm{s} \\
& \text { DF20/21 = } 14 \text { messages/s } \\
& \text { DF0 }=11 \text { messages } / \mathrm{s} \\
& \text { DF17 extended squitter }=4 \text { messages } / \mathrm{s}
\end{aligned}
$$

This implies that total short messages per second are 34 and total long messages per second are 16. If we compare this result with minimum reply rate Mode $\mathrm{S}$ transponder capability specified in ICAO Annex 10, we can see that we are reaching to the limiting values.

A Mode $\mathrm{S}$ transponder shall be able to generate short Mode $S$ replies at the rate of 50 Mode $S$ replies in any one second interval and in addition to any downlink (a transponder level 2, 3 and 4), shall be able to generate at least 16 long replies of 50 Mode $\mathrm{S}$ replies in any one second interval.

We should note that the presented sample represent peak transmission load but on the other hand such results are nothing exceptional in European high traffic density areas. Moreover due to continual traffic growth it could be hardly expected that mode $\mathrm{S}$ load will be decreasing during a foreseeable future.

\section{HYBRID SURVEILLANCE}

Since a significant level of interrogations and replies fall to ACAS operation as you can see on Fig. 2, thus methods for reducing ACAS interrogation rates would offer great benefit to usage of the spectrum. Over time, ACAS standards have been enhanced to provide mechanisms to reduce interrogation rates. Last activities in this area were focused on technique known as Hybrid Surveillance.

Hybrid surveillance takes advantage of $1090 \mathrm{MHz}$ extended squitter to reduce ACAS interrogations. For aircraft at surveillance range, whose announced position pass through the validation test, the position is further tracked by mean of passive acquisition of extended squitter. The validation test mentioned above includes comparison of relative range, relative bearing and barometric altitude determined by the active ACAS interrogations with derived relative range, bearing and altitude from information reported in squitter. If this test is satisfied then an active interrogation to validate the passive position occurs once every 60 seconds.

When the intruder is a near threat in altitude or range, but not both, it is tracked with passive surveillance, and that passive position is actively interrogated once every 10 seconds for validation. Exact conditions are specified below [3]:

$|a| \leq 10000 f t$ AND $\left\{\left(|a| \leq 3000 f t\right.\right.$ OR $\left.\frac{|a-3000 f t|}{|\dot{a}|} \leq 60 s\right)$ OR $\left(r \leq 3 N M\right.$ OR $\left.\left.\frac{r-3 N M}{|\dot{r}|} \leq 60 s\right)\right\}$

Where: $\quad \mathrm{a}=$ intruder altitude separation in $\mathrm{ft}$

$\dot{\mathrm{a}}=$ altitude rate in $\mathrm{ft} / \mathrm{s}$

$\mathrm{r}=$ intruder slant range in $\mathrm{NM}$

$\dot{\mathrm{r}}=$ range rate in $\mathrm{NM} / \mathrm{s}$

When the intruder is a near threat in altitude and range, it is tracked with active surveillance at the standard once per second interrogation rate. If the following conditions are met, full active surveillance occurs [3]:

$|a| \leq 10000 f t$ AND $\left\{\left(|a| \leq 3000 f t\right.\right.$ OR $\left.\frac{|a-3000 f t|}{|\dot{a}|} \leq 60 s\right)$ AND $\left(r \leq 3 N M\right.$ OR $\left.\left.\frac{r-3 N M}{|\dot{r}|} \leq 60 s\right)\right\}$

This basic concept of utilizing passive positions information transmitting within extended squitter (1090ES) provides a means of reducing 1030/1090 MHz activities.

Nevertheless researches have identified inefficiencies within the algorithm presented above as far as RF band consumption is concerned. ACAS without hybrid surveillance interrogates non thread target once every 5 seconds which elicit a short reply and potential threat target once every second by means of short interrogations and replies. Whereas validation interrogations (transmitted once every $10 \mathrm{~s}$ ) during hybrid surveillance elicit long reply messages which includes extended squitter position register. This model was designed by reason of minimize processing effort for real time interpolation to be done by ACAS unit. The acquirement of position based on active measurement and position announced within 1090ES in the same time enable simpler comparison tasks.

Since the length of a validation reply is twice the length of standard ACAS reply, a target that is tracked passively and validated once every 10 seconds doesn't provide reduction in $1090 \mathrm{MHz}$ channel utilization relative to a target that is tracked actively once every 5 seconds [7].

The considered solution is simply use of interrogations which elicit short replies for the effect of subsequent more difficult computational process.

Additional considered improvement could be achieved by means of exclusive use of $1090 \mathrm{ES}$ for surveillance of aircraft meeting certain conditions. This technique is sometimes called as Extended Hybrid Surveillance

The targets that meet the following conditions could be surveyed without any active interrogations [1]: 
A) ADS-B data satisfied quality metrics

B) $-74 \mathrm{dBm} \leq$ Squitter Power $\leq$ Ext. Hybrid Surv. MTL

Quality metrics are based on parameters contained within 1090 ES messages. There are: NIC (Navigation Integrity Category), NAC (Navigation Accuracy Category), SIL (Source Integrity Level), SDA (System Design Assurance). Current draft requirements require a track to be Version 2 extended squitter with $\mathrm{NIC} \geq 6(<0.6 \mathrm{NM}), \mathrm{NAC}_{\mathrm{P}} \geq 7(<0.1 \mathrm{NM})$, $\mathrm{SIL}=3$ and SDA $=2$ or $3[1]$.

Many other ACAS improvements are suggested as far as the $1090 \mathrm{MHz}$ channel load reduction is concerned. For example, significant restriction of ACAS interrogating (reduce interrogating power by $10 \mathrm{~dB}$ ) when is aircraft operating on the airport surface. Or modify validating rate and prevent early transition to active surveillance because of a validating reply is not received.

\section{Phase ENCOCED PULSES IN $1090 \mathrm{MHz}$ REPLIES}

The present specification in ICAO Annex 10 determines that the reply data block shall consist of 56 or 112 data bits formed by binary pulse position modulation encoding of the reply data. Besides that a new standard is developed by ICAO based on phase modulation type reply. This technique is primary intended for $1090 \mathrm{MHz}$ Extended Squitter messages where it could offer increased data capacity.

The technique consists of phase encoded pulses within a single standard pulse associated with a single phase state for each PPM data bit and in each of the four preamble pulses. For phase modulated signals, the PPM modulated reply data shall additionally contain 8PSK modulated data within the bit interval containing the pulse, either the first half or the second half of the interval. (i.e. 3 additional bits within one standard pulse are encoded by means 8PSK modulation). This encoding affords three additional 112-bit message segments within the standard 1090ES transmission. The phase overlay has the potential to increase data throughput, while maintaining backward compatibility to existing 1090 ES transmissions, without the need to increase the Extended Squitter rate.

\section{CONCLUSION}

Although the EUROCONTROL measurements were performed in high penetrated Mode $\mathrm{S}$ area, Mode A/C reply rate still remains relatively high. TCAS activities (especially
$\mathrm{DF}=0$ ) and All Call replies are the two main sources of Mode $\mathrm{S}$ RF pollution on 1090 in Europe. It is need to carefully predict future use of this link and development of new technologies and applications based on usage 1030/1090 RF band. For example, this is very important from the point of view the TIS-B (Traffic Information Service - Broadcast) implementation.

\section{REFERENCES}

[1] ICAO, AERONAUTICAL SURVEILLANCE PANEL (ASP) TECHNICAL SUBGROUP (TSG) MEETING, "Investigation of Spectrum Issues Related to Hybrid Surveillance" [online]. Paris, France, June 2012. Available from <http://adsb.tc.faa.gov/ICAO-TSG/TSG24Paris-2012/ASP TSGWP13-08-Investigation of Spectrum Issues Related to Hybrid Surveillance.pdf>

[2] ICAO, AERONAUTICAL SURVEILLANCE PANEL (ASP), 12th WORKING GROUP MEETING, "Report on 1090 RF Measurements in Europe" [online], prepared by Eric Potier, April 2012. Available from <http://adsb.tc.faa.gov/ICAO-ASP/ASP12-Montreal-April-2012.htm>

[3] ICAO, Annex 10 to the Convention on International Civil Aviation "Aeronautical Telecommunications", Volume IV: "Surveillance Radar and Collision Avoidance Systems". Included Amendment no 85.

[4] ICAO, AERONAUTICAL SURVEILLANCE PANEL (ASP), TECHNICAL SUBGROUP (TSG) MEETING, "Proposal for Initial Draft SARPs Framework for the Specification of the Phase Modulation Technique" [online], Prepared by: Tom Pagano and John Paranzino, January 2012. Available from < http://adsb.tc.faa.gov/ICAOTSG/TSG23-Ft.Lauderdale-2012/ASP TSG WP12-01-Draft SARPs Phase Overlay Transmit.pdf>

[5] ICAO, AERONAUTICAL SURVEILLANCE PANEL (ASP), TECHNICAL SUBGROUP (TSG) MEETING, "The Impacct on Transponder Occupancy Generated by Surveillance System in General" [online], Prepared and presented by Steffen Marquard, January 2012. Available from <http://adsb.tc.faa.gov/ICAO-TSG/TSG23Ft.Lauderdale-2012.htm>

[6] ICAO ASP TSG, "Example of Mode S Transponder activity measured in Europe" [online], February 2009. Available from <http://adsb.tc.faa.gov/ICAO-TSG/TSG17-Ft.Lauderdale-2009/ASP TSG WP06-14 Example of Mode S Transponder Activity Measured in Europe.pdf >

[7] Rose, C. E.; Elder, T. R.; "TCAS surveillance algorithm modification for reduced channel utilization", Digital Avionics Systems Conference (DASC), 2011 IEEE/AIAA 30th ,pp.4E1-1-4E1-7, 16-20 Oct. 2011. ISBN: 978-1-61284-797-9

[8] ICAO, "Surveillance Roadmap" [online], Twelfth Air Navigation Conference, Montréal, 19 to 30 November 2012. Available from $<$ http://www.icao.int/Meetings/anconf12/IPs/ANConf.12.IP.004.1.1.en.p df $>$ 